Pure Appl. Chem., Vol. 83, No. 1, pp. 243-252, 2011.

doi:10.1351/PAC-CON-10-11-04

(c) 2010 IUPAC, Publication date (Web): 6 December 2010

\title{
Integration of surface science, nanoscience, and catalysis*
}

\author{
Cun Wen, Yi Liu, and Franklin (Feng) Tao \\ Department of Chemistry and Biochemistry, University of Notre Dame, \\ Notre Dame, IN 46556, USA
}

\begin{abstract}
This article briefly reviews the development of surface science and its close relevance to nanoscience and heterogeneous catalysis. The focus of this article is to highlight the importance of nanoscale surface science for understanding heterogeneous catalysis performing at solid-gas and solid-liquid interfaces. Surface science has built a foundation for the understanding of catalysis based on the studies of well-defined single-crystal catalysts in the past several decades. Studies of catalysis on well-defined nanoparticles (NPs) significantly promoted the understanding of catalytic mechanisms to an unprecedented level in the last decade. To understand reactions performed on catalytic active sites at nano or atomic scales and thus reach the goal of catalysis by design, studies of the surface of nanocatalysts are crucial. The challenges in such studies are discussed.
\end{abstract}

Keywords: heterogeneous catalysis; interfaces; nanoparticles; nanoscience; surface science.

\section{INTRODUCTION}

Heterogeneous catalysis is in fact the foundation of industrial production [1-3]. More than one-third of the production processes in all industries involve heterogeneous catalysis in their production chains [4,5]. Particularly, it has been the core technology of the chemical industry, oil refineries, conversion of sustainable energy sources, and environmental remediation for several decades.

The application of heterogeneous catalysis to industrial production was realized without understanding of catalytic mechanisms at the microscopic level several decades ago. In that time, people mostly cared about the activity of catalysts and productivity of the production processes using catalysts. Most of the catalysts used at that time were developed on the basis of trial-and-error; lately, the accumulated information from experience, including effects of different support oxides, promotion of alkali metal in reduction of metallic oxide, preparation routes, and pretreatment conditions (gas, temperature, and pressure), did help increase activity, improve selectivity, and enhance stability of catalysts. However, to make an active catalyst consistently needs more information about catalysts and requires the mechanistic understanding of catalysts at the microscopic level at least (Fig. 1). This is particularly important for the design of catalysts with high selectivity since a sustainable green environment directly benefits from application of catalysts with high selectivity in industrial production.

Having benefitted from the development of ultrahigh vacuum (UHV) technology in the 1960s and the followed surface analytical techniques, extensive model studies of heterogeneous catalysis on single crystals of metal catalysts have been performed in the last four decades [1,2]. From the point of view

\footnotetext{
*Pure Appl. Chem. 83, 1-252 (2011). A collection of invited, peer-reviewed articles by former winners of the IUPAC Prize for Young Chemists, in celebration of the International Year of Chemistry 2011.

¥Corresponding author: E-mail: ftao@nd.edu
} 

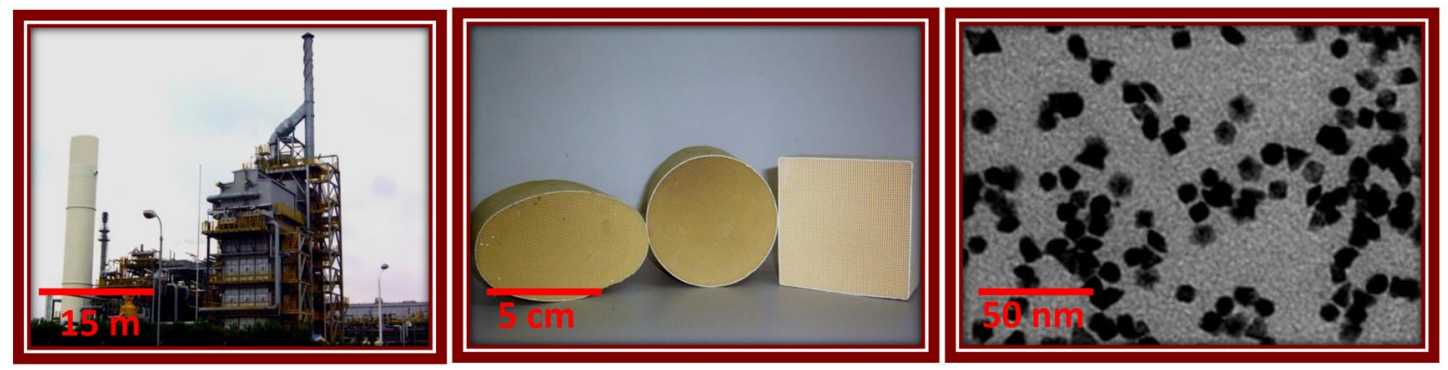

Fig. 1 Vision of heterogeneous catalysis from large-scale reactor to nanoscale catalysts. (a) Picture of industrial reactors; (b) Picture of catalysts with a size of 5-10 cm; (c) Image of nanocatalysts with crystallite size of $10 \mathrm{~nm}$.

of model studies on single-crystal catalysts, a tremendous amount of information about surface structure, adsorption, and molecular desorption was obtained, which built the foundation for understanding industrial catalysis.

Typically, the model catalysts of single crystals used in research labs have a crystalline size of $1 \mathrm{~cm}$. Such a surface has homogeneous domains with a size larger than $100 \mathrm{~nm}$. In contrast, the majority of the industrial catalysts consist of particles in the size range of one to a few hundred nanometers with different size and shape dispersed on support (mostly oxide) or incanted in porous materials $[4,5]$. Catalytic reaction takes place on the active sites of the heterogeneous catalysts. An active site consists of a few or more atoms of surfaces of one or two components of a catalyst. These components are generally metals, oxides, or both. In contrast with the well-defined surface structure of model catalysts of single crystals, nanoparticle (NP) catalysts typically have much more complicated surface structures.

Definitely, an active site is at nanoscale, though different sites have different atomic packing and composition. There could be size-dependent structural and electronic factors on the surface of catalysts with crystallite at nanometer range. These factors could result in size-dependent surface activity and selectivity of nanocatalysts. A classic example of such dependence would be the Au catalysts. A bulk Au metal is generally considered to be catalytically inert, but Au NPs with a size of $1.5-3 \mathrm{~nm}$ deposited on $\mathrm{TiO}_{2}$ are highly active in many processes, such as $\mathrm{CO}$ oxidation [5-9]. Another example is the sizedependent catalytic activity of Rh NPs in the range of $2-12 \mathrm{~nm}$ for CO oxidation [10]. There are examples for size-dependent catalysis induced by size-dependent surface chemistry. Obviously, without understanding of the nanoscale active sites and surface chemistry on catalysts at nanoscale, the rational design of catalyst would be extremely challenging [11].

\section{BRIEF DESCRIPTION OF THE DEVELOPMENT OF SURFACE SCIENCE}

Historically, surface science has been developed since the spontaneous spreading of oil on water was studied by Benjamin Franklin [12]. From the 1900s to 1950s, surface science studies focused on the properties of chemisorbed monolayers, adsorption isotherms, molecular adsorption and dissociation, and energy exchange $[13,14]$. As surface science became important for understanding the production processes using catalysts in industries such as pretreatment, activation, poison, and deactivation of catalysts in production, it has become one of the major areas of chemistry and physics.

In the 1950s, surface science received an opportunity to develop and had an explosive growth driven by the development of UHV technology and the available solid-state-device-based electronics with acceptable cost $[15,16]$. Thus, many efforts were made to the studies of surface structure and chemistry since high-purity and highly ordered single-crystal surfaces could be prepared in UHV in that time. In the 1960s, the advance of surface analytical techniques resulted in a remarkable development of surface science. Many surface phenomena such as adsorption and bonding, oxidation, and catalysis were studied at the molecular level. 
In the 1980s, the invention of various scanning probe microscopes greatly accelerated the development of surface science [17], because these probing techniques make it possible to study surfaces and interfaces at the atomic level. Particularly importantly, these techniques allow scientists to visualize the surface at the atomic level and identify surface structural and electronic features with the highest resolution. This breakthrough moved scientists' vision for materials surface from average information of a large-scale surface to local information of the surface at nano and atomic scales. Numerous surface phenomena were re-examined at the atomic level. Many great details at the atomic level on the surface were revealed. For example, scanning tunneling microscopy (STM) gave us an opportunity to visualize atoms on various surfaces of metals and semiconductors [18,19]. Atomic level information achieved with these techniques significantly aided identification of specific catalytic site of catalytic reactions [20,21]. In addition, the breakthrough of the surface analytical techniques expanded the territory of surface science to almost all areas of materials science, physics, chemistry, and mechanical and electronic engineering. For example, semiconductor and microelectronic industries have largely benefitted from the advancement of surface science [22-26], as all the protocols for the fabrication of semiconductor devices and microelectronic components extensively involved surface science and technology.

In recent decades, the explosive development of bioscience gives surface science another opportunity $[27,28]$. Such a development offers surface science the third explosive growth because the studies of various bioprocesses and biofunctions performed in nature largely rely on the understanding of the complicated liquid-liquid, liquid-solid, and liquid-gas interfacial phenomena in these biosystems. For example, the functions of some biospecies largely depend on the self-assembly of specific biomolecules at interfaces in nature. The terms "biosurface" and "biointerface" have been widely used to describe these studies.

\section{IMPORTANCE OF SURFACE SCIENCE}

The term "surface science" often makes people instantly have a connection to various surface analytical techniques used in many fields of chemistry, materials science, and physics. It is true that the development of surface science has significantly relied on the invention and advance of surface analytical techniques [1,2]. In fact, every aspect of our daily life and work involves surface science. Most of the production processes in chemical industries involve heterogeneous catalysis to different extents, which are performed on the surface of solid catalysts under high pressure of reactants and high temperature of catalysts. Oil refining relies on chemical reactions at interfaces of gas feedstock and various catalysts. New energy conversion processes extensively involve heterogeneous catalysis such as (1) evolution of $\mathrm{H}_{2}$ and $\mathrm{O}_{2}$ on the surfaces of cocatalysts in solar-driven water-splitting [29-34], and (2) generation of electricity from oxidation of fuel molecules on the surface of the electrode in fuel cells [35-37]. Most of the issues in environmental science involve the chemical process occurring on the surface of various materials such as minerals under ambient conditions [38-40]. For example, chemical conversion of greenhouse gases to fuel and conversion of poisonous emission are typically heterogeneous processes occurring on some specific catalysts $[41,42]$.

\section{SURFACE SCIENCE: DEALING WITH CHEMISTRY AT THE INTERFACE OF TWO PHASES}

Generally, the interactions at the interface of two different phases can be categorized into noncovalent and covalent binding. Corresponding to this categorization, strategies used in the design of new materials can be categorized as (1) molecular self-assembly through weak noncovalent forces, and (2) molecular breaking of chemical bonds and the formation of new ones $[23,43,44]$. The macroscopic selfassembled structure formed on a substrate is typically held together by various weak noncovalent forces between adsorbed molecules within a self-assembled structure and between the adsorbed molecules and template at the nanoscale. In this case, ordered supramolecular systems with new structure and property

() 2010, IUPAC

Pure Appl. Chem., Vol. 83, No. 1, pp. 243-252, 2011 
form spontaneously from the original components. With weak noncovalent binding including electrostatic interactions between static molecular charges, hydrogen bonding, van der Waals forces, $\pi-\pi$ interactions, hydrophilic binding, and charge-transfer interactions, many new self-assembled structures with various sizes, shapes, and functions have been designed [23,43,44]. Different from weak interactions in these systems, strong chemical bonding is commonly existent in many interfacial systems such as functionalization of functionalized semiconductor surface with organic molecules and chemically coating layers resisting surface corrosion [23,43,45]. A large number of surface phenomena result from or at least involve the strong chemical binding at interfaces. For example, surface etching, chemisorption, and thin-film growth extensively rely on the formation of chemical bonds at interfaces.

Other than the strong chemical bonding and weak van de Waals binding, chemical adsorption of molecules on metal surfaces in heterogeneous catalysis can be considered as the third type of interaction $[1,2,46]$. The strength of this type of interaction is between the weak van der Waals and strong chemical binding (mostly, covalent bonding). Such a binding with a medium strength is in fact necessary for heterogeneous catalysis since (1) a binding of reactant molecules with certain strength makes sure certain residence time of reactant molecules on the surfaces of catalysts, which potentially allows the buildup of a high coverage at high pressure, and aids bond breaking in some cases, and (2) a too strong binding will, however, decrease molecular mobility on surfaces, which is necessary to produce intermediates or product molecules.

\section{SURFACE SCIENCE: CLOSELY RELEVANT TO NANOSCIENCE}

Surface science had been studied at nanoscale before the "nano" term was frequently used in the recent two decades. Surface processes are performed at nanoscale even on the surface of a particle in the range of a centimeter or even larger. Information volume along the surface normal is definitely in the range of nanometers since interaction on surfaces is only performed in the surface region with a thickness of a few atomic layers. In addition, STM revealed that actually most of the samples with size at macroscopic level are heterogeneous in lateral dimension. Typically, the uniform surface feature is only identified at tens to one hundred of nanometers. Thus, surface processes do occur at nano scale even the material has macroscopic level. For crystallite with a size less than $100 \mathrm{~nm}$, such as different types of $0 \mathrm{D}, 1 \mathrm{D}, 2 \mathrm{D}$, and 3D nanomaterials, certainly the surface chemistry on these materials is already at nanoscale. Studies of chemistry on the surface at nanoscale are important for understanding chemical and even physical properties of nanomaterials. Thus, we call the surface chemistry on nanomaterials or the nanoscale domain of macroscopic materials "nanoscale surface science".

As schematically shown in Fig. 2, several geometric structural factors are size-dependent. These size-dependent geometric structural factors can induce size-dependent electronic factors, surface chemistry, and functions of nanomaterials. With the decrease of nanomaterials size, the fraction of all atoms at the edge of two faces and corners is increased. The increased fraction of atoms on surface results in large surface free energy. Moreover, the fraction of atoms directly interacting with atoms of support is increased with the decrease of size. In addition, the packing of atoms on surface and in the surface region of nanomaterials could not follow the crystallographic periodicity of atomic packing of materials with a macroscopic size (Fig. 3). Since surface free energy is increased, density of defects on surface is increased. These size-dependent structural features on the surface of nanomaterials are expected to induce size-dependent reactivity of nanomaterials.

Precise control of the nanoscale surface chemistry and physics could aid size and shape control in the synthesis of nanomaterials [47-59]. Different binding between surfactant molecules and metal atoms on the surface of NPs could facilitate the production of monodispersed NPs. A preferential binding of surfactant molecules on a specific face could direct the synthesis of nanomaterials with specific shapes $[47,60,61]$. Using poly(vinylpyrrolidone) with certain molecular weight helps the synthesis of $\mathrm{Rh}, \mathrm{Pt}$, and Pd NPs with specific size. By adding a second capping agent, trimethyl(tetradecyl) ammonium bromide (TTAB), the strong binding of $\mathrm{Br}^{-}$can stabilize the $\{100\}$ faces of these metal NPs and 


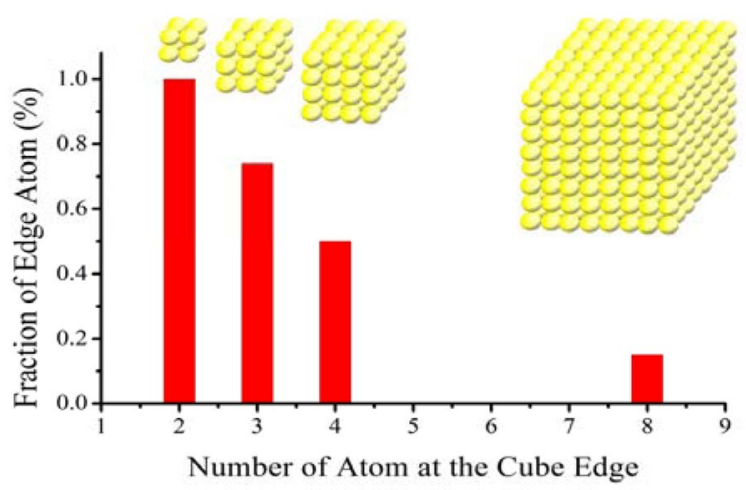

Fig. 2 Schematic of nanomaterials with different sizes.
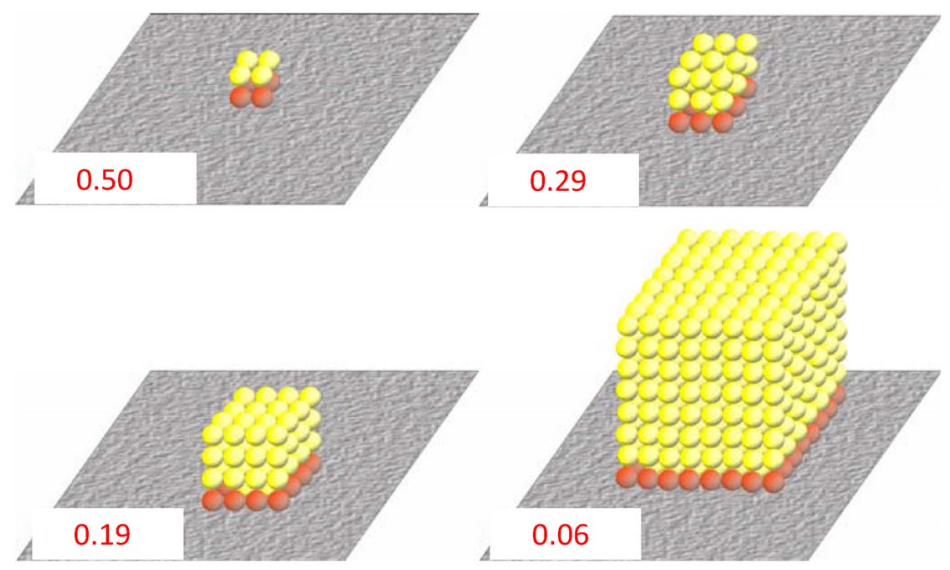

Fig. 3 Size-dependent oxide-metal interfacial area of catalysts. The atomic fraction of metal atoms (red) at oxide/metal interface is listed at the bottom left of each panel.

thus produce uniform cubic NPs. Obviously, understanding the surface chemistry and binding of capping molecules on the metal surface of NPs would definitely promote a precise synthesis of nanomaterials.

Studies of the nanoscale surface of materials and devices are essential for understanding the function of materials and devices. Self-assembly of organic molecules to form organic thin films on different substrates is an important strategy for modification of the chemical and physical properties of the solid surface. It is one of the main approaches for functionalization of solid surfaces as the properties and functions of the attached organic layers are generally absent for inorganic substrates. More importantly, these organic modification and functionalization allow surface and interfacial properties to be tailored controllably, since myriad organic molecules are available and the structure and property of organic materials can be systematically varied. This advantage is based on the principle that the surface and interfacial structures determine surface properties and functions. Figure 4 schematically shows the molecular packing on Au surface. The orientations of ending group $\left(-\mathrm{CH}_{2}-\mathrm{CH}_{3}\right)$ are different, depending on the number (odd or even) of carbon atoms in the chain. In addition, if the ending group is replaced by a functional group with large size, the binding between organic layer and $\mathrm{Au}(111)$ can be modified. More importantly, the slightly different binding at molecular level could result in a measureable difference in chemical, physical, and interfacial properties. Some of these properties change as the number of carbon atoms in the molecular chain. These tunable properties include wettability, surface 


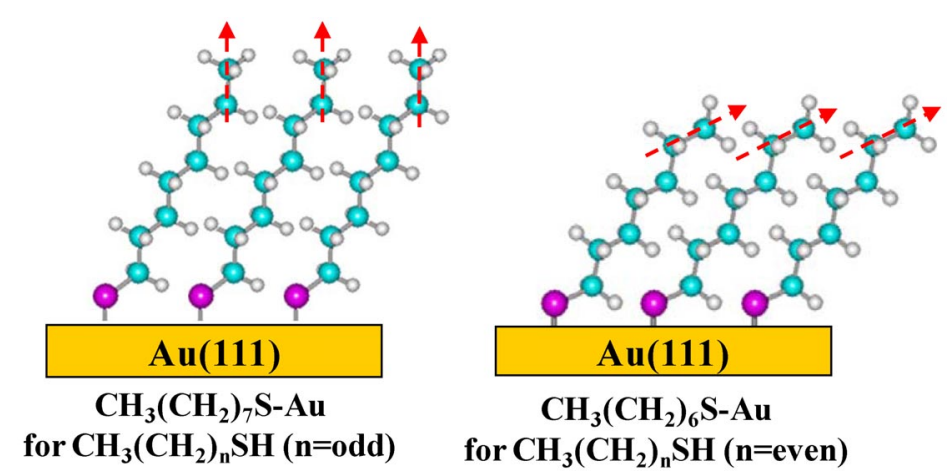

Fig. 4 Schematics of different binding geometries of organic molecules with odd and even number of $\mathrm{CH}_{2}$ units assembled on $\mathrm{Au}(111)$.

work function, adhesion, surface exchange kinetics, electron transfer, electrochemical properties, and chemical reactivity [43].

The nanoscale surface/interface science of the nanomaterials and nanodevices includes surface structure, binding configuration, molecular ordering, interfacial interaction of heterogeneous phases via various noncovalent interactions or strong chemical bonds, and surface and interfacial properties such as conductivity, surface polarity, and friction. Thus, how deeply we understand the nanoscale surface and interface of heterogeneous materials and devices largely imparts whether we can successfully synthesize nanomaterials and creatively design nanodevices for specific requirements. The necessity and importance of understanding nanoscale surface science in the creation of various heterogeneous materials and devices gives an unprecedented chance and challenge for the development of surface science.

\section{CATALYSIS AT NANOSCALE}

Catalysis occurs on active sites. An active site typically consists of a few or even more atoms of the surface of the active phase of a catalyst particle or entity. The active phase could be one component such as metal or two components such as metal and oxide. The process of molecular catalysis is essentially performed at the nano or atomic level owing to the localized active sites. In fact, the size of a crystallite of industrial catalysts is in the range of a few to a few hundred nanometers [1,2]. Heterogeneous catalysts are nanomaterials indeed. But scientists and engineers in the catalytic community did not use the term "nano" a few decades ago.

As the size of the crystallite of a catalyst decreases, a few size-dependent structural and electronic factors appear, which potentially leads to size- and structure-dependent catalytic activity and selectivity. With the decrease of size, the fraction of atoms with low coordination number (the atoms at edge, corner, and defect sites) is increased (Fig. 2). In most cases, these under-coordinated sites are catalytically active [62-66]; typically, they are the active sites for bond-breaking to form intermediates or products. With the decrease of size, the actual fraction of metal atoms binding to support materials is increased as schematically shown in Fig. 3; therefore, size-dependent interfacial effect is expected. It was illustrated by a recent work published by Bao et al. [67]. In addition, owing to increased surface relaxation, the packing of metal atoms on and in the surface region of catalysts at nanoscale could not follow the crystallography implemented in macroscopic crystals (100 $\mathrm{nm}$ or larger). This was demonstrated by a restructuring of terraces on a stepped surface with a width of $1.3 \mathrm{~nm}$ in contrast to the preservation of structuring of surface with large size (100 $\mathrm{nm}$ or larger) [19,66]. In addition, the binding configurations of reactant molecules on the surface at nanoscale could be different from those (three-fold, bridge, and on-top) on large domains of model catalysts of single crystals. 
These size-dependent structural factors do result in size-dependent surface chemistry at the nanoscale and thus size-dependent catalytic activity. One of the recent examples showed with the decrease of size of Rh NPs, Rh atoms of the surface layers could be oxidized easily [10]. A different fraction of surface oxides formed on Rh NPs with different sizes results in different catalytic activity for $\mathrm{CO}$ oxidation.

\section{FUTURE CHALLENGES}

The above discussion has indicated that the studies of nanoscale surface chemistry of catalysts are crucial for understanding the reaction mechanism on catalysts for the goal of catalysis by design. However, there is still a long way to go before the rational design of industrial catalysts. Some of the existing challenges are briefly described in the following paragraphs:

(a) Increased complexity of surface and interfaces of real catalysts in contrast to model catalysts Although the model catalysts investigated in recent years are closer to realistic catalysts compared to the well-defined single-crystal catalyst, it still could not represent the complexity of realistic catalysts. Most of the industrial catalysts consist of metal and oxide components. In many of these cases, oxide and metal components of a catalysts work synergistically [68-79]. It is necessary to explore the surface and interface of oxide-metal components in catalysts. However, obviously these surfaces and interfaces are complicated in contrast to single-component model catalysts.

(b) Challenges in synthesis of nanomaterials

New synthetic strategies are required to generate nanostructures with specific sites predicted by theoretical studies or inspired by experimental results. An example is that the high Miller-Index facets often have very active sites which are generally desired for high catalytic activity for some reactions according to the understanding from surface science research. However, it is very difficult to synthesize NPs with high Miller-Index facets. Recently, Wang et al. [51] synthesized Pt NPs with high Miller-Index facets with a creative electrochemical method. Thus, sophisticated material synthetic methods are important to apply the fundamental understanding or surface science to catalysis studies. In fact, there are many challenges including how to synthesize oxide-metal nanocomposite catalysts with well-controlled interfacial structures.

(c) Challenges in characterization techniques

The importance of advanced characterization techniques have been briefly discussed above. The development of characterization techniques has greatly promoted the development of surface science during the past several decades. For example, traditional nuclear magnetic resonance spectroscopy (NMR) could not distinctly discern different structures on the surface of solid catalyst. Nevertheless, Kwak et al. [80] unraveled this problem by using NMR with ultrahigh magnetic field. Their results clearly indicated that the surface pentacoordinate $\mathrm{Al}^{3+}$ sites were important to anchor Pt. To attain information on nanoscale surface of a catalyst for understanding catalytic mechanism, the advanced characterization techniques are needed since a few challenges exist for the analysis of the surface of nanocatalysts [81]. One challenge is characterization of nanoscale surfaces buried in the cavity of porous materials and at hidden oxide-metal interfaces. Another challenge is the characterization of surface chemistry of nanocatalysts in the size range of $1 \mathrm{~nm}$ or less since the surface of such a small entity is extremely sensitive to any incident radiation including photo, electron, and ion beams. New nondestructive analysis of NPs at nanometer range is highly desirable. 


\section{ACKNOWLEDGMENT}

We acknowledge the financial support from the Department of Chemistry and Biochemistry, College of Science, Sustainable Energy Initiative, Office of Research at the University of Notre Dame.

\section{REFERENCES}

1. G. A. Somorjai, Y. Li. Introduction to Surface Chemistry and Catalysis, $2^{\text {nd }}$ ed., John Wiley, Hoboken (2010).

2. G. Ertl, H. Knozinger, J. Weitkamp. Handbook of Heterogeneous Catalysis, Wiley-VCH, Weinheim (2008).

3. C. H. Bartholomew, R. J. Farrauto. Fundamentals of Industrial Catalytic Processes, $2^{\text {nd }}$ ed., John Wiley, New York (2005).

4. A. T. Bell. Science 299, 1688 (2003).

5. D. R. Rolison. Science 299, 1698 (2003).

6. M. S. Chen, D. W. Goodman. Science 306, 252 (2004).

7. M. S. Chen, D. W. Goodman. Acc. Chem. Res. 39, 739 (2006).

8. G. J. Hutchings. Dalton Trans. 41, 5523 (2008).

9. L. C. Grabow, M. Mavrikakis. Angew. Chem., Int. Ed. 47, 7390 (2008).

10. M. E. Grass, Y. W. Zhang, D. R. Butcher, J. Y. Park, Y. M. Li, H. Bluhm, K. M. Bratlie, T. F. Zhang, G. A. Somorjai. Angew. Chem., Int. Ed. 47, 8893 (2008).

11. G. J. Hutchings. J. Mater. Chem. 19, 1222 (2009).

12. Philos. Trans. R. Soc. London 64, 445 (1774).

13. N. Nilius. Surf. Sci. Rep. 64, 595 (2009).

14. T. T. Tsong. Prog. Surf. Sci. 64, 199 (2000).

15. G. Ertl, H.-J. Freund. Phys. Today 52, 32 (1999).

16. R. R. Chianelli, G. Berhault, B. Torres. Catal. Today 147, 275 (2009).

17. G. Binnig, H. Rohrer, C. Gerber, E. Weibel. Phys. Rev. Lett. 49, 57 (1982).

18. F. Besenbacher, J. V. Lauritsen, T. R. Linderoth, E. Lægsgaard, R. T. Vang, S. Wendt. Surf. Sci. 603, 1315 (2009).

19. R. T. Vang, J. V. Lauritsen, E. Lægsgaard, F. Besenbacher. Chem. Soc. Rev. 37, 2191 (2008).

20. R. Otero, F. Rosei, F. Besenbacher. Аnпи. Rev. Phys. Chem. 57, 497 (2006).

21. J. V. Lauritsen, F. Besenbacher. In Advances in Catalysis, Vol. 50, p. 97, Academic Press, New York (2006).

22. H. Neergaard Waltenburg, J. T. Yates. Chem. Rev. 95, 1589 (1995).

23. F. Tao, S. L. Bernasek, G.-Q. Xu. Chem. Rev. 109, 3991 (2009).

24. D. Stiévenard, B. Legrand. Prog. Surf. Sci. 81, 112 (2006).

25. M. A. Filler, S. F. Bent. Prog. Surf. Sci. 73, 1 (2003).

26. R. A. Wolkow. Annu. Rev. Phys. Chem. 50, 413 (1999).

27. L. M. Demers, D. S. Ginger, S.-J. Park, Z. Li, S.-W. Chung, C. A. Mirkin. Science 296, 1836 (2002).

28. T. A. Taton, C. A. Mirkin, R. L. Letsinger. Science 289, 1757 (2000).

29. P. V. Kamat. J. Phys. Chem. Lett. 1, 520 (2009).

30. P. V. Kamat. J. Phys. Chem. C 112, 18737 (2008).

31. P. V. Kamat. J. Phys. Chem. C 111, 2834 (2007).

32. V. Chakrapani, K. Tvrdy, P. V. Kamat. J. Am. Chem. Soc. 132, 1228 (2010).

33. B. Farrow, P. V. Kamat. J. Am. Chem. Soc. 131, 11124 (2009).

34. K. Maeda, K. Domen. Chem. Mater. 22, 612 (2009).

35. R. Adzic, J. Zhang, K. Sasaki, M. Vukmirovic, M. Shao, J. Wang, A. Nilekar, M. Mavrikakis, J. Valerio, F. Uribe. Top. Catal. 46, 249 (2007). 
36. Y. Shao-Horn, W. Sheng, S. Chen, P. Ferreira, E. Holby, D. Morgan. Top. Catal. 46, 285 (2007).

37. Y. H. Bing, H. S. Liu, L. Zhang, D. Ghosh, J. J. Zhang. Chem. Soc. Rev. 39, 2184 (2010).

38. G. Centi, P. Ciambelli, S. Perathoner, P. Russo. Catal. Today 75, 3 (2002).

39. G. Oberdorster, E. Oberdorster, J. Oberdorster. Environ. Health Perspect. 113, 823 (2005).

40. W. Weiss, W. Ranke. Prog. Surf. Sci. 70, 1 (2002).

41. X. M. Liu, G. Q. Lu, Z. F. Yan, J. Beltramini. Ind. Eng. Chem. Res. 42, 6518 (2003).

42. M. Bowker. Chem. Soc. Rev. 37, 2204 (2008).

43. F. Tao, S. L. Bernasek. Chem. Rev. 107, 1408 (2007).

44. J. C. Love, L. A. Estroff, J. K. Kriebel, R. G. Nuzzo, G. M. Whitesides. Chem. Rev. 105, 1103 (2005).

45. Z. Ma, F. Zaera. Surf. Sci. Rep. 61, 229 (2006).

46. G. Ertl. Reactions at Solid Surface, John Wiley, New York (2009).

47. A. R. Tao, S. Habas, P. Yang. Small 4, 310 (2008).

48. N. Tessler, V. Medvedev, M. Kazes, S. H. Kan, U. Banin. Science 295, 1506 (2002).

49. X. Wang, J. Zhuang, Q. Peng, Y. D. Li. Nature 437, 121 (2005).

50. Z. Y. Zhou, Z. Z. Huang, D. J. Chen, Q. Wang, N. Tian, S. G. Sun. Angew. Chem., Int. Ed. 49, 411 (2010).

51. N. Tian, Z.-Y. Zhou, S.-G. Sun, Y. Ding, Z. L. Wang. Science 316, 732 (2007).

52. M. J. Mulvihill, B. L. Rupert, R. He, A. Hochbaum, J. Arnold, P. Yang. J. Am. Chem. Soc. 127, 16040 (2005).

53. D. Gao, R. He, C. Carraro, R. T. Howe, P. Yang, R. Maboudian. J. Am. Chem. Soc. 127, 4574 (2005).

54. C. Qian, F. Kim, L. Ma, F. Tsui, P. Yang, J. Liu. J. Am. Chem. Soc. 126, 1195 (2004).

55. R. Fan, Y. Wu, D. Li, M. Yue, A. Majumdar, P. Yang. J. Am. Chem. Soc. 125, 5254 (2003).

56. Y. M. Lee, M. A. Garcia, N. A. F. Huls, S. H. Sun. Angew. Chem., Int. Ed. 49, 1271 (2010).

57. Y. G. Sun, C. H. Lei. Angew. Chem., Int. Ed. 48, 6824 (2009).

58. M. Yada, M. Mihara, S. Mouri, M. Kuroki, T. Kijima. Adv. Mater. 14, 309 (2002).

59. C. Wen, Y. Liu, Y. Guo, Y. Wang, G. Lu. Solid State Sci. 11, 1985 (2009).

60. Y. Zhang, M. E. Grass, J. N. Kuhn, F. Tao, S. E. Habas, W. Huang, P. Yang, G. A. Somorjai. J. Am. Chem. Soc. 130, 5868 (2008).

61. Y. Xia, Y. J. Xiong, B. Lim, S. E. Skrabalak. Angew. Chem., Int. Ed. 48, 60 (2009).

62. F. Besenbacher, I. Chorkendorff, B. S. Clausen, B. Hammer, A. M. Molenbroek, J. K. Nørskov, I. Stensgaard. Science 279, 1913 (1998).

63. K. Morgenstern, E. Lægsgaard, F. Besenbacher. Phys. Rev. Lett. 94, 166104 (2005).

64. R. T. Vang, K. Honkala, S. Dahl, E. K. Vestergaard, J. Schnadt, E. Lægsgaard, B. S. Clausen, J. K. Nørskov, F. Besenbacher. Nat. Mater. 4, 160 (2005).

65. S. L. Bernasek, W. J. Siekhaus, G. A. Somorjai. Phys. Rev. Lett. 30, 1202 (1973).

66. F. Tao, S. Dag, L. W. Wang, Z. Liu, D. R. Butcher, H. Bluhm, M. Salmeron, G. A. Somorjai. Science 327, 850 (2010).

67. Q. Fu, W.-X. Li, Y. Yao, H. Liu, H.-Y. Su, D. Ma, X.-K. Gu, L. Chen, Z. Wang, H. Zhang, B. Wang, X. Bao. Science 328, 1141 (2010).

68. J. C. Kang, S. L. Zhang, Q. H. Zhang, Y. Wang. Angew. Chem., Int. Ed. 48, 2565 (2009).

69. E. de Smit, I. Swart, J. F. Creemer, C. Karunakaran, D. Bertwistle, H. W. Zandbergen, F. M. F. de Groot, B. M. Weckhuysen. Angew. Chem., Int. Ed. 48, 3632 (2009).

70. C. X. Xiao, Z. P. Cai, T. Wang, Y. Kou, N. Yan. Angew. Chem., Int. Ed. 47, 746 (2008).

71. M. Shen, F. Zaera. Angew. Chem., Int. Ed. 47, 6583 (2008).

72. G. B. Yu, B. Sun, Y. Pei, S. H. Xie, S. R. Yan, M. H. Qiao, K. N. Fan, X. X. Zhang, B. N. Zong. J. Am. Chem. Soc. 132, 935 (2010).

73. G. L. Bezemer, T. J. Remans, A. P. van Bavel, A. I. Dugulan. J. Am. Chem. Soc. 132, 8540 (2010). 
74. J. A. Rodriguez, J. Graciani, J. Evans, J. B. Park, F. Yang, D. Stacchiola, S. D. Senanayake, S. G. Ma, M. Perez, P. Liu, J. F. Sanz, J. Hrbek. Angew. Chem., Int. Ed. 48, 8047 (2009).

75. Q. Fu, H. Saltsburg, M. Flytzani-Stephanopoulos. Science 301, 935 (2003).

76. W. L. Deng, M. Flytzani-Stephanopoulos. Angew. Chem., Int. Ed. 45, 2285 (2006).

77. J. Knudsen, A. U. Nilekar, R. T. Vang, J. Schnadt, E. L. Kunkes, J. A. Dumesic, M. Mavrikakis, F. Besenbacher. J. Am. Chem. Soc. 129, 6485 (2007).

78. J. B. Park, J. Graciani, J. Evans, D. Stacchiola, S. D. Senanayake, L. Barrio, P. Liu, J. F. Sanz, J. Hrbek, J. A. Rodriguez. J. Am. Chem. Soc. 132, 356 (2010).

79. J. A. Rodriguez, P. Liu, J. Hrbek, J. Evans, M. Perez. Angew. Chem., Int. Ed. 46, 1329 (2007).

80. J. H. Kwak, J. Hu, D. Mei, C.-W. Yi, D. H. Kim, C. H. F. Peden, L. F. Allard, J. Szanyi. Science 325, 1670 (2009).

81. M. S. Altman. Science 327, 789 (2010). 